\title{
Scheduling the Australian Football League
}

\author{
Jari Kyngäs ${ }^{1}$, Kimmo Nurmi ${ }^{1}$, Nico Kyngäs ${ }^{1}$, George Lilley ${ }^{2}$, Thea Salter ${ }^{3}$ and Dries Goossens ${ }^{4 *}$ \\ ${ }^{1}$ Satakunta University of Applied Sciences, Pori, Finland; ${ }^{2}$ Box Hill Senior Secondary College, Mont Albert, \\ Australia; ${ }^{3}$ AFL House, Docklands, Australia; and ${ }^{4}$ Faculty of Economics and Business Administration, Ghent \\ University, Tweekerkenstraat 2, 9000 Ghent, Belgium
}

Generating a schedule for a professional sports league is an extremely demanding task. Good schedules have many benefits for the league, such as higher attendance and TV viewership, lower costs and increased fairness. The Australian Football League is particularly interesting because of an unusual competition format integrating a single round-robin tournament with additional games. Furthermore, several teams have multiple home venues and some venues are shared by multiple teams. This paper presents a 3-phase process to schedule the Australian Football League. The resulting solution outperforms the official schedule with respect to minimizing and balancing travel distance and breaks, while satisfying more requirements.

Journal of the Operational Research Society (2017) 68(8), 973-982. doi:10.1057/s41274-016-0145-8; published online 6 December 2016

Keywords: sports; timetabling; multiple venues; travel distance; heuristics

\section{Introduction}

Australian Rules football (officially Australian football) was invented in Melbourne, Australia, and first played in 1858 (Blainey, 2010). The history of the Australian Football League (AFL) dates back to 1897, but games have been played in some kind of league format since 1877 . Originally intended to keep the cricketers fit during winter time, Australian football soon became highly popular in Australia and is now practised in large parts of the world. When measured by attendance, it is by far the most popular sport in Australia. The spectator average per match for the season 2013 was 33,500. The most popular matches in the regular season have more than 80,000 spectators.

As have many professional sports leagues, the Australian Football League (AFL) has become a big business. Accordingly, the quality of the schedules has become increasingly important, as the schedule has a direct impact on revenue for all involved parties. For instance, the number of spectators in the stadia and the travelling costs for the teams are influenced by the schedule, and TV networks that pay for broadcasting rights want the most attractive games to be scheduled at commercially interesting times. Scheduling the Australian Football League is a very demanding task. It has in fact been claimed to be the most difficult mathematical problem in world sport by the Herald Sun. ${ }^{1}$ Not surprisingly, the problem

*Correspondence: Dries Goossens, Faculty of Economics and Business Administration, Ghent University, Tweekerkenstraat 2, 9000 Ghent, Belgium. E-mail: dries.goossens@ugent.be

\footnotetext{
1 "Supercomputers tackle toughest mathemathics problem in world sport-the AFL draw". See http://www.news.com.au/sport/afl/super computers-tackle-toughest-mathemathics-problem-in-world-sport-the-afldraw/story-fnelctok-1226500295493 (Published 22.10.2012)
}

of scheduling sport leagues has drawn the attention of an increasing number of researchers. Nurmi et al (2010) report on a growing number of cases in which academic researchers have been able to close a scheduling contract with a professional sports league owner.

In a sports tournament, $n$ teams play against each other over a period of time according to a given timetable. The teams belong to a league, which organizes games or matches between the teams. Each game consists of an ordered pair of teams, denoted $i-j$, where team $i$ plays at home-that is, uses its own venue (stadium) for a game-and team $j$ plays away. In a round-robin tournament, each team plays against every other team a fixed number of times. Most sports leagues play a double round-robin tournament, where the teams meet once at home and once away. Games are grouped in rounds, which are played on one or more consecutive days (usually a weekend). If a team does not play on some round, we say it has a bye on that round. A schedule is compact if it uses the minimum number of rounds required to schedule all the games; otherwise, it is relaxed. If a team plays two home or two away games in two consecutive rounds, it is said to have a break. In general, for reasons of fairness, breaks are to be avoided. However, in order to reduce travelling costs, a team may prefer to have two or more consecutive away games. This could be the case if its stadium is located far from the opponent's venues, and the venues of these opponents are close to each other. A series of consecutive away games is called an away tour. Excellent overviews of sports scheduling can be found in Easton and Nemhauser (2004) and Rasmussen and Trick (2008). An extensive bibliography can 
be found on a website maintained by $\mathrm{Knust}^{2}$ and in an annotated bibliography by Kendall et al (2010).

The scheduling problem faced by the Australian Football League can be seen as a so-called Constrained Sports Scheduling Problem (CSSP). In a CSSP, the goal is to find a feasible solution that is the most acceptable for the sports league owner - that is, a solution that has no hard constraint violations and that minimizes the weighted sum of the soft constraint violations. Several types of constraints that occur frequently in constrained sport scheduling problems have been listed by Nurmi et al (2010). Nevertheless, the AFL scheduling problem has two interesting and relatively novel features. First, the AFL consists of a single round-robin tournament complemented with 5 additional matches for each team. These additional matches are no post-season or play-off competition; instead, they are mixed with the round-robin matches. Integrating additional matches into a round-robin tournament is uncommon, but has been studied before by academics in the context of the New Zealand Rugby Union Cup (Johnston and Wright, 2014) and the Finnish Major Ice Hockey League (Kyngäs and Nurmi, 2009). In these competitions, the opponents and the home advantage for the additional matches are fixed before the schedule is created; in the New Zealand Rugby Union Cup, teams get to pick their opponents for the additional matches in a media-covered selection event. In the AFL, however, deciding the opponents and the home advantage for the additional matches is part of the scheduling process. Furthermore, the league uses a relaxed schedule, such that each team has a bye in one of the three rounds centred around the middle of the season.

A second interesting feature is the fact that some teams in the AFL have multiple home venues. In addition, two stadia, Etihad and MCG, host almost half of all the matches. Half of the teams play one or more home matches at these stadia, and some teems need to play a minimum number of away matches at Etihad Stadium. Furthermore, as the AFL is trying to expand the sport throughout the country and even to New Zealand, some of the matches are played in cities and stadia that do not have a permanent home team. Settings with multiple venues have been studied from a theoretical point of view by, for example, Urban and Russell (2003, 2006), de Werra et al (2006) and Ikebe and Tamura (2008). However, in these contributions, the idea is that the stadia are not linked to any team, and the goal is that each team plays the same number of games in each stadium. We are not aware of any contribution on real-life sport scheduling that deals with multiple home venues. In Section 2, we give a detailed problem description, covering all the requests that need to be taken into account for the 2013 season.

As the AFL scheduling problem turns out too demanding to solve in a single model, we have developed a 3-phase approach. In the first phase, opponents and home advantage are decided, the second phase assigns matches to rounds, and

${ }^{2}$ See http://www.inf.uos.de/knust/sportssched/sportlit_class the final phase decides on the kick-off times and venues. Each of these phases is tackled with an implementation of the PEAST (Population, Ejection, Annealing, Shuffling, Tabu) heuristic, which has proven its value for several other complex real-life problems as, for example, workforce scheduling (Nurmi and Kyngäs, 2011). Details on our solution method are given in Section 3. The AFL currently uses software from the firm "Optimal Planning Solutions" to craft the schedule. This company creates fixtures for leading competitions across the globe including NFL football, European soccer, the NRL Rugby and Super Rugby. Our goal is to improve on the official schedule, in particular with respect to minimizing and balancing travel distance and the number of breaks. In Section 4, we report on our computational results and compare our schedule with the official schedule for the 2013 season.

\section{Problem Description}

The Australian Football League has 18 teams (see Table 1). Figure 1 shows the location of the teams. While the majority of the teams are situated in or around the Victoria region, two of the teams are located remotely in the northeast of the country (Queensland) and two are far away in Western Australia. Figure 1 also depicts the stadia that are used to host the matches. In order to further popularize Australian Football and to create opportunities to attend a game in cities that do not have a home team, the AFL also makes use of stadia in cities as Darwin (Northern Australia), Hobart and Launceston (Tasmania), Cairns (Northern Queensland) and Wellington (New Zealand, which is not depicted in Figure 1). The AFL decides, before the schedule is made, which matches will take place in these stadia, and at what date and time. Furthermore, the big stadia in Melbourne (MCG, Etihad) are also used to host a number of popular games involving home teams that do not have these stadia as their home ground. Moreover, these stadia are shared by several teams as their home venue. Contractual agreements determine the number of matches that are to be played for every stadium.

The AFL competition has a complicated structure. It consists of each team playing against every other team once, i.e. a single round robin. In addition, each team has to play 5 extra matches. This adds up to 22 matches for each team: 11 home and 11 away matches. The integration of a single round robin and additional matches makes the schedule different from most other professional sports league schedules, where additional matches are usually played as a play-off tournament, after the regular round-robin competition. Furthermore, the AFL schedule consists of 23 rounds (i.e. time-relaxed), and each team has one bye during rounds 11-13. These 3 rounds consist of 6 matches, while the other 20 rounds have 9 matches.

The two main objectives are related to travel distance and breaks. Australia is a big country, which causes extensive travel loads for the teams, especially for the teams from Queensland and Western Australia. For instance, in 2013, the 
total travel distance was $243125 \mathrm{~km}$. For a remote team like Fremantle, the official season schedule included nearly $35000 \mathrm{~km}$ of travel, while for any team in Victoria, the total travel was less than $11000 \mathrm{~km}$. One objective in the AFL scheduling problem is to balance total travelling between teams from the same state, without exceeding the current total travel distance. In particular, travel loads from non-Victorian teams should be as equal in length as possible. Therefore, we will measure this balance as the sum of differences in travel distance between each pair of non-Victorian local rivals.

The second objective is to minimize the total number of breaks. Break minimization is quite common as an objective in sport scheduling (see Kendall et al, 2010). In fact, De Werra (1981) has presented an efficient algorithm to compute a

Table 1 Eighteen teams in the AFL and their home venues

\begin{tabular}{ll}
\hline Adelaide Crows (AAMI) & Hawthorn (MCG) \\
Brisbane Lions (Gabba) & Melbourne (MCG) \\
Carlton (MCG, Etihad) & North Melbourne \\
& (Etihad) \\
Collingwood (MCG) & Port Adelaide (AAMI) \\
Essendon (MCG, Etihad) & Richmond (MCG) \\
Fremantle (Patersons) & St Kilda (Etihad) \\
Geelong Cats (Simonds) & Sydney Swans (SCG, \\
& ANZ) \\
Gold Coast Suns (Metricon) & Western Bulldogs \\
& (Etihad) \\
Greater Western Sydney Giants & West Coast Eagles \\
(Skoda, Manuka) & (Patersons) \\
\hline
\end{tabular}

minimum break schedule for a single round-robin tournament without further constraints. Apart from the total number of breaks, the AFL also wants to minimize the number of consecutive breaks for each team (this occurs when a team plays 3 home games or 3 away games in a row), as well as the number of breaks for each team. With respect to the latter, 5 breaks or less per team is considered reasonable.

Achieving these objectives is further complicated by an extensive list of constraints that need to be taken into account, communicated to us by the league authorities. We have grouped these constraints into 3 categories, related to home teams and opponents (Section 2.1), order of the matches (Section 2.2), and venues and kick-off times (Section 2.3).

\subsection{Home Teams and Opponents}

The teams play a single round robin with five additional matches. Therefore, each team meets five teams twice (home and away) and twelve teams only once during the season. The schedule should secure fairness for the teams. It should also increase revenues for owners/shareholders by increasing the number of spectators and decreasing travelling costs. Finally, it should secure the interest of media, TV network and fans and optimize matches considering their needs. The problem is to schedule the home advantage and opponents such that all teams play under similar conditions. For instance, the home advantage should be set such that all teams have to play a minimum of five matches in Victoria. Furthermore, the five

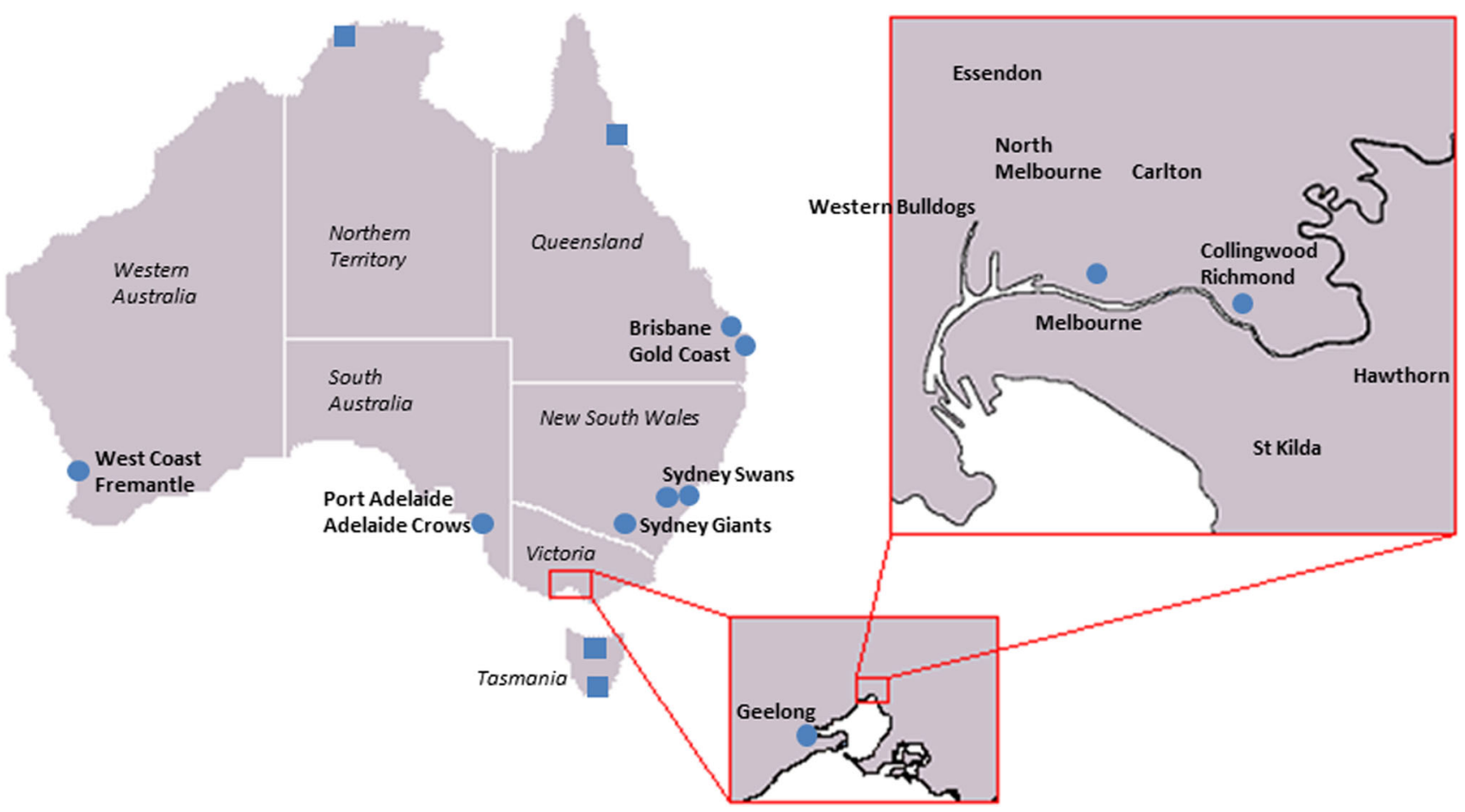

Figure 1 Eighteen AFL teams. Dots represent home venues; squares correspond to stadia without a permanent home team (New Zealand is not depicted). 
additional matches should be such that all local rivals and "big" clubs should meet each other twice. In total, the league authorities defined 11 selection rules, which we converted to equally many hard constraints.

The home advantage of the matches is subject to the following constraints:

1. All teams have to play a minimum of 5 matches in Victoria.

2. Victorian teams should travel outside Victoria a minimum of 5 times.

3. Each team must have at least one home match against Collingwood or Essendon.

4. Each team has to travel to Western Australia at least once.

5. For a number of matches, the home advantage is fixed.

The selection of the opponents in the 5 additional matches for each team is as follows:

6. Blockbuster matches (i.e. between top teams) must be included.

7. Matches between local rivals (Adelaide Crows and Port Adelaide, Brisbane Lions and Gold Coast Suns, Fremantle and West Coast Eagles, Greater Western Sydney Giants and Sydney Swans) must be included.

8. The top four teams from the previous season can have only one meeting with the bottom four teams from the previous season, with the exception of the Sydney rivals.

9. The top eight teams from the previous season should play at least three other top eight teams twice

10. The bottom ten teams from the previous season should play at least three other bottom ten teams twice.

11. The bottom two teams from the previous season should not meet the top eight teams from the previous season twice (Sydney rivals are an exception).

Matches are defined as "blockbuster matches" by the league authorities, in consultation with the broadcasters. They are a subset of the matches featuring two of the big six teams from Victoria (i.e. Carlton, Collingwood, Essendon, Geelong Cats, Hawthorn and Richmond). Note that some of these constraints may be conflicting (e.g. depending on last season's ranking). If this is the case, the league authorities make exceptions or slight alterations to these rules (for instance, in 2013, Collingwood and Richmond were not required to be matched up twice).

\subsection{Order of the Matches}

Matches are grouped in rounds, which have an order. The season consists of 23 rounds: nine matches can be assigned to rounds $1-10$ and $14-23$, and 6 matches to rounds $11-13$. The requirements concerning the order of the matches and grouping in rounds can be described with the following nine constraints.

12. Pre-assigned matches, i.e. for which the round is fixed, must be respected
13. There must be at least 6 rounds between two matches with the same opponents.

14. There should be at most 1 home match per round for each of four pairs of teams. The pairs are Adelaide Crows and Port Adelaide, Brisbane Lions and Gold Coast Suns, Fremantle and West Coast Eagles, Greater Western Sydney Giants and Sydney Swans.

15. Non-Victorian teams that travelled in round 23 in the previous season cannot travel in round 23 this season.

16. If two teams play against each other twice, the second match cannot be played before round 11 .

17. If two teams play against each other only once, this match cannot be played on the final round.

18. There must be at least 6 rounds between visits to Western Australia

19. There must be at least 6 rounds between visits to Queensland

20. Geelong Cats must play exactly 4 home matches in the first 10 rounds.

The number of matches for which the round has been fixed beforehand may vary from year to year; in the 2013 season, there were 42 of these matches. Constraint (14) enforces that local rivals never play at home in the same round. Constraint (20) is due to venue redevelopment and at the request of Geelong Cats; it does not apply in other seasons.

\subsection{Venues and Kick-Off Times}

This group of constraints deals with the exact weekdays and kick-off times, and the venues of the matches. A regular round consists of 9 matches, of which 1 is played on Friday, 5 are played on Saturday and 3 are played on Sunday. In rounds 11-13, there are only 6 matches and the distribution of matches is 1 match on Friday, 3 matches on Saturday and 2 matches on Sunday. Moreover, in rounds 1, 7 and 10, one Sunday match is actually played on Monday.

Most of the constraints related to kick-off times originate from broadcasters (all AFL matches are broadcast on television). Foxtel is an Australian pay television company that produces and broadcasts five matches a week (in a regular round)-three on Saturday and two on Sunday. The Seven Network broadcasts the remaining four matches (in a regular round) on a free-to-air network. It is important that the broadcasts do not overlap on Saturday or Sunday in Western Australia, Southern Australia, Queensland and New South Wales. This is applicable to the Adelaide and Port Adelaide teams, the Fremantle and West Coast Eagles teams, the Brisbane Lions and Gold Coast Suns teams, and also to the Sydney Swans and GWS Giants teams. This is manageable because there are at least 3 days on which to play, and subsequently there are also varying timeslots to schedule within each day so they can be scheduled on the same day in a different timeslot (e.g. afternoon and night). 
Australian Football is a very physical game, with a relatively high injury rate. In order to give the players a chance to recover from minor injuries, a break of at least 6 days between consecutive matches of a team is mandatory. Anzac Day (25 April) is an exception because no matter which weekday it happens to be, at least one match is played, including a match between Essendon and Collingwood. All the teams playing on Anzac Day must be prepared to play either their preceding or following match with a shorter break. Of course, the schedule should be made in such a way that it places these teams' preceding and following matches as far away from Anzac Day as possible.

Two stadia, Etihad and MCG, host almost half of all the matches (93 out of 198) and should mostly be used for Friday matches. Furthermore, half of the teams play home matches at these stadia. Two of these teams play the majority of their home matches at Etihad Stadium, and the remaining play a varying number of home matches at both stadia. Of the latter, two teams have to play a minimum number of away matches at Etihad Stadium. Confidentiality prevents us from disclosing exactly how many (and in some cases also which) matches should be played at each stadium, but these requirements are crucial for the league organizers.

This results in the following constraints:

21. (There should be a minimum of 6 days between each match (with exceptions resulting from Anzac Day).

22. There should be no matches in overlapping broadcasting slots, so that all local matches can be broadcast on freeto-air in each market.

23. All clubs have to play at least one match at the MCG stadium.

24. There should be a minimum of 45 matches in MCG stadium.

25. There should be a minimum of 48 matches in Etihad stadium.

26. Other venue contractual requirements (pre-defined number of matches played at each stadium).

27. At least 15 matches in the Etihad or MCG stadium should be played on Friday.

28. No day or twilight matches at TIO stadium

29. No Sunday early or Saturday afternoon matches at Patersons stadium.

30. No home matches for Geelong Cats at Simonds stadium until Round 10.

Note that for the pre-assigned matches (12), the kick-off time and venue has also been fixed by the AFL authorities, prior to the scheduling process. Constraint (30) is also due to venue redevelopment at Simons stadium and does not apply in other seasons.

\section{Solution Method}

Without any constraints other than that each teams plays against each other team the same number of times, and no teams plays more than once per round, creating a schedule is easy (see, e.g. De Werra, 1981). However, as soon as additional constraints such as stadium availability have to be taken into account, the problem becomes NP-hard (Easton and Nemhauser, 2004), and no constant-factor approximation algorithm exists unless $\mathrm{P}=\mathrm{NP}$ (Briskorn et al, 2010). Therefore, our approach involves decomposing the sports scheduling problem into subproblems, which are computationally more manageable and solved sequentially.

A phased approach is quite common in sport scheduling. Most papers apply a so-called first break, then schedule approach, where in a first phase, the home advantage is settled for each team in each round, and in a second phase, the opponents are decided (e.g. Nemhauser and Trick, 1998). In settings where breaks are less important, a "first schedule, then break" approach, which settles the opponents in each round before the home advantage is decided, is typically used (e.g. Trick, 2001). Our approach is different in the sense that in our first phase, we decide on the opponents as well as the home advantage. However, the assignment of matches to rounds is only handled in the second phase. A third phase settles the weekday, kick-off, time and venue for each match.

\subsection{A 3-Phased Approach}

We build a schedule by solving 3 phases consecutively, where the outcome of each phase is the input for the next. In the first phase, the opponents in the 11 home matches are determined for each team. Notice that as a result, the five additional matches are settled as well. In this phase, we take into account constraints (1)-(11), which are all related to opponents and home advantage (see Section 2.1). Furthermore, in order to balance the travel distance over the teams, we enforce that all teams travel 2 or 3 times to either Western Australia or Queensland (of course, only for teams not from Western Australia or Queensland). Including this constraint in phase 1 somewhat equalizes the travel load between the teams. Without this restriction, some teams might visit Western Australia once and Queensland not at all, while some other teams might make two visits to each of these areas. The second phase includes assigning a round for each match. Clearly, in this phase we take into account constraints (12)-(20), which involve the order and grouping of the matches (see Section 2.2). The third and final phase settles the weekday and kick-off time, as well as the venue for each match, taking into account constraints (21)-(30) as discussed in Section 2.3.

The success of a phased approach depends on the importance and complexity of the constraints and objectives handled in each phase. Ideally, the more difficult and important a constraint or objective is, the earlier in the phased approach it should be dealt with. The first phase deals with all constraints that are essential with respect to fairness of the schedule and attractiveness of the fixtures. Furthermore, travel distance is mostly determined by setting the opponents in the additional 
games and the home advantage (phase 1). In other sports [e.g. Major League Baseball (Easton and Nemhauser, 2001)], travel costs are minimized by creating away tours. In the AFL, this is not option: given that a break of at least six days between consecutive matches of a team is mandatory, teams travel back to their home city after every match. Hence, the order of the rounds (phase 2) is of little importance with respect to travel distance. Although the third phase determines the home venues, its impact on travel distance is also limited. Indeed, the matches to be played in the remote cities without a permanent home team (e.g. Darwin) are all pre-fixed by contract. Furthermore, if a team has multiple home venues, they tend to be close to each other. For instance, the MCG and Etihad stadium are less than $5 \mathrm{~km}$ apart. Hence, in general, a good solution for phase 1 offers interesting prospects for high-quality schedule, if feasibility issues in the subsequent phases can be avoided.

Phase 2 determines the second objective: the number of breaks, and how they are balanced over the teams. With respect to the constraints, it handles the important, but relatively easy requirement that local rivals should not play home games on the same round, as well as a number of fairness constraints that are considered less crucial by the AFL authorities. In the third phase, the two heavily used stadia Etihad and MCG might at first sight seem to cause a feasibility issue. However, this is not the case in practice. Because of the local rivals, who can never play a home game in the same round (phase 1), there are always 4 matches played in stadia other than Etihad and MCG. This leaves a maximum of five matches to be played at Etihad, MCG and/or Simonds Stadium, which can easily be handled in a weekend. Constraint (30), which forbids home matches for Geelong Cats at their home venue (Simonds stadium) until Round 10, is another example of a tough constraint in phase 3. Again, we anticipate possible feasibility issues earlier in the decision process: constraint (20) in phase 2 limits the number of home matches for Geelong Cats in the first 10 rounds. Eventually, phase 3 has never turned out infeasible in any of our test runs.

\subsection{The PEAST Algorithm}

In this section, we discuss the PEAST algorithm, which was used to solve all of the three phases described in Section 3.1. The PEAST algorithm is a heuristic, which has been implemented to optimize several types of real-world scheduling problems and is in industrial use. The PEAST algorithm is in essence a population-based local search method; the pseudocode of the algorithm is given in Figure 2. Population-based methods use a population of solutions in each iteration, which enables them to explore a wide range of promising areas in the search space. At the same time, they are highly suited to escape from local optima. Our algorithm is a variant of the cooperative local search introduced by Preux and Talbi (1999). In a cooperative local search scheme, each individual carries out its own local search, in our case the greedy hill-climbing mutation (GHCM) heuristic.
Input the population size $n$, the iteration limit $t$, the cloning interval $c$, the shuffling interval $s$ and the ADAGEN update interval $a$ Generate a random initial population of schedules $S_{\text {f }}$ for $i=1, \ldots, n$ Set best_S $=$ null and iteration $=1$

WHILE iteration $\leq t$

$k=1$

WHILE $k \leq n$

(explore promising areas in the search space) Apply GHCM to schedule $S_{k}$ to get a new schedule IF $\operatorname{Cost}\left(S_{k}\right)<$ Cost(best_S) THEN Set best_S $=S_{k}$ $k=k+1$

END REPEAT

(avoid staying stuck in the promising search areas too long)

Update the simulated annealing framework

IF iteration $\equiv 0(\bmod c)$ THEN

(favor the best schedule, i.e. use elitism)

Replace the worst schedule with the best one

IF iteration $\equiv 0(\bmod s)$ THEN

(escape from the local optimum)

Apply shuffling operators

IF iteration $\equiv 0(\bmod a)$ THEN

Update the ADAGEN framework

iteration $=$ iteration +1

END WHILE

Output best_S

Figure 2 Pseudo-code of the PEAST algorithm.

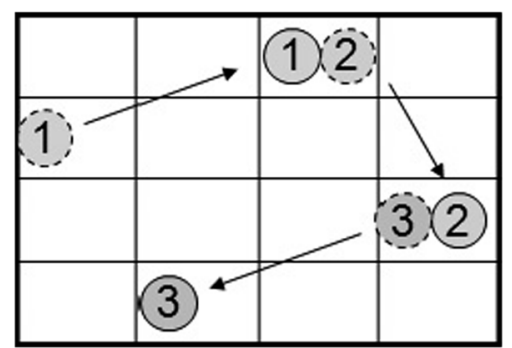

Figure 3 A sequence of moves in the GHCM heuristic.

The GHCM operator explores promising areas in the search space by extending the basic hill-climbing step to generate a sequence of moves in one step, leading from one solution to another. The operator is based on ideas similar to the LinKernighan procedures (Lin and Kernighan, 1973) and ejection chains (Glover, 1992). It moves an object, $o_{1}$, from its old position, $p_{1}$, to a new position, $p_{2}$, and then moves another object, $o_{2}$, from position $p_{2}$ to a new position, $p_{3}$, and so on, ending up with a sequence of moves. Picture the positions as cells as shown in Figure 3. The initial object selection is random, using tournament selection. The cell that receives the object is selected by considering all the possible cells and selecting the one that causes the least increase in the objective function when only considering the relocation cost. Then, another object from that cell is selected by considering all the objects in that cell and picking the one for which the removal causes the biggest decrease in the objective function when only considering the removal cost. Next, a new cell for that object is selected, and so on. The sequence of moves stops if the last move causes an increase in the objective function value and the value is larger than that of the previous non-improving move, or if the maximum number of moves is reached. 
In the first phase, the GHCM framework has a position for each of the 11 home games for each team. The objects that are being moved around correspond with the opponents (i.e. the away teams) in these matches. In the second phase, a position is a round and there is an object for each match determined in phase 1 . In the third phase, there is a position for each allowable (timeslot, venue)-pair. The objects are again the matches.

A new cell for an object in GHCM is selected by evaluating all possible cells and greedily selecting the best one. Furthermore, in every $c$ th iteration, the least fit schedule in the population is replaced with a clone of the fittest individual. This operation is completely irrespective of the globally fittest schedule (best_S in Figure 2) found by that time in the search process. A greedy approach combined with elitism implies the risk of staying stuck in the same areas of the search space (i.e. the objective function value does not improve for some predefined number of generations). We counter this by using tabu search and a refined simulated annealing method. A tabu list (Glover et al, 1985) is used to prevent reverse order moves in a single application of the GHCM operator. The simulated annealing refinement is used to decide whether or not to commit to a sequence of moves in the GHCM operator. This refinement is different from the standard simulated annealing (Kirkpatrick et al, 1983). It is used on three occasions: (1) when choosing an object to be moved, (2) when choosing the destination of the object and (3) when the sequence of moves is cut short (a worsening move is made, and it worsens the solution more than the previous worsening move did). A detailed discussion of the tabu search and simulated annealing refinement can be found in Kyngäs et al (2012).

The PEAST algorithm applies a number of shuffling operators to perturb a solution into a potentially worse solution in order to escape from local optima. The idea of shuffling is the same as in hyper-heuristics (Burke et al, 2013) but used with the opposite intention. Hyper-heuristic is a mechanism that chooses a heuristic from a set of simple heuristics, applies it to the current solution to get a better solution, then chooses another heuristic and applies it, and continues this iterative cycle until the termination criterion is satisfied. We introduce a number of simple heuristics that are used to worsen the current solution instead of improving it. We used the following five shuffling operators: (1) 1-5 random moves, e.g. moving a match from a random round to another random round, (2) swapping the rounds of 1-5 games, (3) moving 1-all games from a round to random rounds, (4) swapping the home advantage between 1 and 5 pairs of games and (5) swapping all the games in two random rounds.

The AFL problem is a multi-objective optimization problem, i.e. a problem where multiple objective functions have to be optimized simultaneously. The objective functions usually compete in such a way that improving one objective function value most likely decreases the other objective function values. In the PEAST algorithm, the objective functions are considered as soft constraints, while constraints (1)-(29) are hard constraints. Traditional penalty methods assign positive weights (penalties) to the soft constraints and sum the violation scores to the hard constraint values to get a single value to be optimized. The PEAST algorithm uses the an adaptive genetic penalty method (ADAGEN), which assigns dynamic weights to the hard constraints based on the search trajectory and the constant weights assigned to the soft constraints, according to their significance. The significance is given by the problem owner. This means that we are searching for a solution that minimizes the following (penalty) function:

$$
\sum_{i} \alpha_{i} f_{i}(x)+\sum_{i} c_{i} g_{i}(x)
$$

where $\alpha_{i}=$ a dynamically adjusted weight for hard constraint $i$; $f_{\mathrm{i}}(x)=$ cost of violations of hard constraint $i ; c_{\mathrm{i}}=$ a fixed weight for soft constraint $i ; g_{\mathrm{i}}(x)=$ cost of violations of soft constraint $i$.

The weights of the hard constraint are repeatedly updated after a fixed number of generations using the method given in by Nurmi (1998).

The PEAST algorithm uses random initial solutions. In our extensive test runs, we have found no evidence that sophisticated initial solutions significantly improve our results. On the contrary, random initial solutions tend to yield superior or at least as good results. Even though the best parameter values vary depending on the problem and the instance, our experience with the PEAST algorithm has shown that the following values can safely be used in different real-world problems and instances:

- The population size is 10 .

- The cloning interval is 500 .

- The shuffling interval is 5000 .

- The maximum length of the move sequence in the GHCM operator is 10 .

- The size of the tournament selection is 7 .

- The length of the tabu list is 10 , which equals the length of the move sequence.

- In the simulated annealing framework, we stop the cooling at some pre-defined temperature. Therefore, after a certain number of iterations, $m$, we continue to accept an increase in the cost function with some constant probability. We choose $m$ equal to $t / 2$, where $t$ is the maximum number of iterations and $p$ is equal to 0.0001 .

We are aware of the fact that we have used many different heuristic methods in the PEAST algorithm. The acronym PEAST stems from the methods used: Population, Ejection, Annealing, Shuffling and Tabu. One might think that the outcome is nothing more than a collection of old ideas. However, to the best of our knowledge, the heart of the algorithm, the GHCM operator, is one of a kind. The same applies to our implementation of the shuffling operators, simulated annealing and the ADAGEN penalty method. A tabu list improves the efficiency of the GHCM operator considerably. 
Table 2 Comparison between the official schedule and our schedule in terms of constraints (violated constraints are italicized)

\begin{tabular}{|c|c|c|}
\hline Constraints & Official schedule & Our schedule \\
\hline (1) All clubs play a minimum of 5 matches in Victoria & $5-7$ & $6-7$ \\
\hline (2) Victoria teams travel maximum of six times & $\mathrm{Ok}$ & $\mathrm{Ok}$ \\
\hline (3) Each team has at least one home match against Essendon or Collingwood & $\mathrm{Ok}$ & $\mathrm{Ok}$ \\
\hline (4) Each team travels to Western Australia once & $\mathrm{Ok}$ & $\mathrm{Ok}$ \\
\hline (5) For a number of matches, the home advantage is fixed & $\mathrm{Ok}$ & $\mathrm{Ok}$ \\
\hline (6) Blockbuster matches included in additional matches & $\mathrm{Ok}$ & $\mathrm{Ok}$ \\
\hline (7) Local derbies included in additional matches & Ok & $\mathrm{Ok}$ \\
\hline (8) Top 4 teams do not play against bottom 4 teams twice-exception Sydney Rivals & $\begin{array}{l}\text { Western Bulldogs versus } \\
\text { Adelaide }\end{array}$ & $O k$ \\
\hline (9) Top 8 teams play at least 3 other top 8 teams twice & $\begin{array}{l}\text { One top } 8 \text { team plays only } 2 \text { top } \\
8 \text { team twice }\end{array}$ & $O k$ \\
\hline (10) Bottom 10 teams play at least 3 other bottom 10 teams twice & Ok & Ok \\
\hline (11) Bottom 2 teams do not play top 8 teams twice-exception Sydney Rivals & $\mathrm{Ok}$ & $\mathrm{Ok}$ \\
\hline (12) Pre-assigned matches & $\mathrm{Ok}$ & $\mathrm{Ok}$ \\
\hline (13) Must be a minimum of 6 rounds between two matches with the same opponents & $\mathrm{Ok}$ & $\mathrm{Ok}$ \\
\hline (14) Local rivals never playing home in same round & Ok & $\mathrm{Ok}$ \\
\hline (15) Alternate travel between non-Vic teams in round 23 & $\mathrm{Ok}$ & Ok \\
\hline (16) No teams meet for the second time before round 11 & Ok & Ok \\
\hline (17) All teams must play each other once by round 22 & $\mathrm{Ok}$ & $\mathrm{Ok}$ \\
\hline (18) At least 6 rounds between visits to Western Australia & 3 times 5 rounds gap & $\begin{array}{l}1 \text { time } 5 \\
\text { rounds gap }\end{array}$ \\
\hline (19) At least 6 rounds between visits to Queensland & 1 time 2 rounds gap & 2 times 4 \\
\hline (20) Geelong Cats must play exactly 4 home matches in the first 10 rounds & Ok & Ok \\
\hline (21) Minimum 6-day break between each match, with exceptions for Anzac Day & $\mathrm{Ok}$ & $\mathrm{Ok}$ \\
\hline $\begin{array}{l}\text { (22) No matches in overlapping broadcasting slots, so that all local matches can be } \\
\text { broadcast on free-to-air in each market. }\end{array}$ & Ok & Ok \\
\hline (23) All teams to play at least one match at MCG & Ok & Ok \\
\hline (24) Minimum of 45 matches in MCG & $\mathrm{Ok}$ & $\mathrm{Ok}$ \\
\hline (25) Minimum of 48 matches in Etihad & Ok & $\mathrm{Ok}$ \\
\hline (26) Other venue contractual requirements & $\mathrm{Ok}$ & $\mathrm{Ok}$ \\
\hline (27) Number of Friday matches at MCG or Etihad & 15 & 17 \\
\hline (28) No day or twilight matches at TIO Stadium & Ok & Ok \\
\hline (29) No Sunday early or Saturday afternoon matches at Patersons Stadium & $\mathrm{Ok}$ & $\mathrm{Ok}$ \\
\hline (30) No home matches for Geelong Cats at Simonds Stadium until round 10 & Ok & Ok \\
\hline
\end{tabular}

\section{Computational Results}

In this section, we compare our schedule to the official schedule for the 2013 season. $^{3}$ Tables 2 and 3 summarize the comparison with respect to the constraints and objectives, respectively. The requirements and objectives were provided to us by the league authorities, and we also received feedback from them on our optimization model. The comparison shows that according to the criteria communicated to us, our solution is better than the official schedule, which was also acknowledged by the league authorities.

Table 2 lists the constraints, grouped as discussed in Section 2 . In the official schedule, 4 constraints were violated. Adelaide, which was a top 4 team in 2012, was paired up with bottom team Western Bulldogs twice, and Fremantle only met 2 other top 8 teams from 2012 twice. Both violations were avoided in our schedule. In fact, we implemented a more strict version of

${ }^{3}$ The official 2013 schedule can be found at http://en.wikipedia.org/wiki/ 2013_AFL_season; our schedule is available upon request from the authors. constraint (9) in our model-that is, "exactly three times"because we think it would be unfair if a team had to meet the top eight teams more than three times (as was the case in the official schedule). We were able to find such a tightened solution. None of the schedules succeeded in having at least 6 rounds between long-distance travels to Western Australia and Queensland. Nevertheless, in our schedule it occurs just once that a team has only 5 rounds between visits to Western Australia (compared to 3 times in the official schedule). For travels to Queensland, two teams have an interval of only 4 rounds in our schedule. However, this still seems better than the official schedule, which features 1 team with 5 rounds and another with merely 2 rounds in between. Finally, we point out that although the official schedule satisfies the constraint of having at least 15 Friday matches at MCG or Etihad, our schedule outperforms the official schedule with 17 Friday matches in these stadia.

Our results with respect to the two objectives, travel distance and number of breaks are given in Table 3 . The most significant difference is the sum of differences in travel distance between each pair of non-Victorian local rivals. This shows that travel 
Table 3 Comparison between the official schedule and our schedule in terms of objectives (travel distance and break related)

\begin{tabular}{lll}
\hline Objectives & Official schedule & Our schedule \\
\hline The sum of differences of the total travelling of non-Victoria teams & 9474 & 632 \\
Total travel distance & 243125 & 160208 \\
Total travelling of non-Victoria teams & 82917 & 153558 \\
Total travelling of Victoria teams & GC 18347 & 86607 \\
Total travelling of Gold Coast and Brisbane Lions & BL 21614 & GC 18347 \\
Total travelling of Adelaide and Port Adelaide & PA 11176 & BL 18347 \\
& A 13126 & PA 11776 \\
Total travelling of West Coast Eagles and Fremantle & WCE 33601 & F 11850 \\
& SS 12243 & WCE 34101 \\
Total travelling of Sydney Swans and GWS & GWS 15300 & GWS 12243 \\
Range of total travelling for Victoria teams & $6718-10992$ & SS 12800 \\
Total number of breaks & 94 & $6711-10987$ \\
Number of 3 breaks at home & 2 & 74 \\
Number of 3 breaks away & 3 & 2 \\
Number of breaks per team (exceeding five) & $8:$ four times & $7:$ once \\
\end{tabular}

distance is far better balanced in our schedule, which is also illustrated by the pairwise comparison of travel distance for local rivals in Table 3. Additionally, we managed to reduce the total travel distance, with a little over $1 \%$. Table 3 also shows that we reduced travelling for the heavily burdened nonVictoria teams, at the expense of the centrally located teams. Balancing the travelling distance of local teams most certainly increases overall satisfaction. Our solution also has considerably fewer breaks: 74 instead of 94 in the official schedule. Another improvement is that no team has 3 away games in a row; we were not able to avoid two series of 3 consecutive home games though. Furthermore, the breaks are better balanced over the teams. We only have four teams that exceed 5 breaks, the number which is considered reasonable by the AFL authorities; only one team has 8 breaks. Overall, we managed to reduce the number of breaks for 9 teams; no team ended up with more breaks than in the official schedule.

\section{Conclusions}

Scheduling the Australian Football League (AFL) is a challenging task. The format of the AFL fixture is quite different compared to other professional sports leagues. One of the most interesting features is that it includes a single round robin with 18 teams, integrated with 5 additional matches per team. These extra matches should be selected in a way that the league can offer attractive games at commercially interesting times, however without bringing too much imbalance with respect to fairness and travel cost between the teams. Another remarkable feature is the fact that some teams have multiple home venues, and at the same time, stadia are the home venue for multiple teams. Indeed, two stadia host almost half of all the matches, and half of the teams play home matches at these stadia.
Travelling is a big issue for the teams. The travelling distance ranges from $6000 \mathrm{~km}$ up to nearly $35,000 \mathrm{~km}$ per season per team. These are big numbers, considering only 11 away matches are played during the season. Another concern is reducing and balancing the number of breaks.

Like many other studies of complex real-life problems, the fact that our results are based on a single problem instance is a drawback that forces us to formulate conclusions with due caution. Nevertheless, we showed that a phased approach is capable of producing a high-quality solution for the 2013 AFL scheduling problem. Each phase in this approach was solved using the PEAST algorithm, which turned out quite flexible to handle the various constraints in each phase. Although the AFL already made use of a renowned private company to optimize their schedule, our approach was able to offer significant improvements in terms of reducing and balancing travel costs as well as the number of breaks. At the same time, we managed to satisfy more constraints than the official schedule. Despite these results and the fact that the league authorities recognize the superiority of our schedule, at the time of writing, we have not been able to come to an agreement to schedule future seasons of the AFL.

Each year there is a lot of debate in the media about the quality and fairness of the schedule. For instance, experts claimed that Hawthorn had a very rough start to the 2013 season because they played against strong teams in the beginning of the season. They also pointed out that Hawthorn had to face five of the eight strongest teams from the previous year twice. Nevertheless, Hawthorn ended up as number one at the end of the season. So, although we were able to find a fairer schedule for Hawthorn, it seems they would not have needed it. The reduction and improved balance of the travel costs, however, is more tangible. 


\section{References}

Blainey G (2010). A Game of Our Own-The Origins of Australian Football. Black Inc., Australia, pp. 7.

Briskorn D, Drexl A and Spieksma FCR (2010). Round robin tournaments and three index assignments. 4OR A Quarterly Journal of Operations Research 8(4):365-374.

Burke EK, Gendreau M, Hyde M, Kendall G, Ochoa G, Özcan E and Qu R (2013). Hyper-heuristics: a survey of the state of the art. Journal of the Operational Research Society 64(12):1695-1724.

de Werra D, Ekim T and Raess C (2006). Construction of sports schedules with multiple venues. Discrete Applied Mathematics 154(1):47-58.

de Werra D (1981). Scheduling in sports. In: Hansen, P., (editor) Studies on Graphs and Discrete Programming, North Holland, pp 381-395.

Easton K, Nemhauser G and Trick M (2001). The travelling tournament problem: description and benchmarks. In: Proceedings of the 7th International Conference on Principles and Practice of Constraint Programming, Lecture Notes in Computer Science, vol 2239, pp 580-584.

Easton K, Nemhauser G and Trick M (2004). Sports scheduling. In: Leung (editor) Handbook of Scheduling. CRC Press, Florida, USA, pp. 52.1-52.19.

Glover F, McMillan C and Novick B (1985). Interactive decision software and computer graphics for architectural and space planning. Annals of Operations Research 5(3):557-573.

Glover F (1992). New ejection chain and alternating path methods for travelling salesman problems. In: Sharda, Balci and Zenios (editors), Computer Science and Operations Research: New Developments in Their Interfaces. Elsevier, pp. 449-509.

Ikebe YT and Tamura A (2008). On the existence of sports schedules with multiple venues. Discrete Applied Mathematics 156(10): $1694-1710$

Johnston M and Wright M (2014). Prior analysis and scheduling of the 2011 Rugby Union ITM Cup in New Zealand. Journal of the Operational Research Society 65(8):1292-1300.

Kendall G, Knust S, Ribeiro CC and Urrutia S (2010). Scheduling in sports: an annotated bibliography. Computers and Operations Research 37(1):1-19.

Kirkpatrick S, Gelatt CD Jr and Vecchi MP (1983). Optimization by simulated annealing. Science 220(4598):671-680.

Kyngäs N, Goossens D, Nurmi K, Kyngäs J (2012). Optimizing the unlimited shift generation problem. In: Di Chio C, Agapitos A,
Cagnoni S, Cotta C and Fernandez de Vega F (editors) Applications of Evolutionary Computation, Lecture Notes in Computer Science, vol 7248, pp 508-518.

Kyngäs J and Nurmi K (2009). Scheduling the finnish major ice hockey league. In: Proceedings of the IEEE Symposium on Computational Intelligence in Scheduling (CI-Sched), Nashville (USA), pp. 84-89.

Lin S and Kernighan BW (1973). An effective heuristic for the travelling salesman problem. Operations Research 21(2):498-516.

Nemhauser GL and Trick MA (1998). Scheduling a major college basketball conference. Operations Research 46(1):1-8.

Nurmi K (1998). Genetic Algorithms for Timetabling and Travelling Salesman Problems, Ph.D. dissertation, Dept. of Applied Mathematics, University of Turku, Finland.

Nurmi K, Goossens D, Bartsch T, Bonomo F, Briskorn D, Duran G, Kyngäs J, Marenco J, Ribeiro CC, Spieksma FCR, Urrutia S and Wolf-Yadlin R (2010). A framework for scheduling professional sports leagues. In: Ao S-I, Castillo O, Douglas C, Feng DD and Lee J-A (editors) IAENG Transactions on Engineering Technologies. Lecture Notes in Engineering and Computer Science 2182: 1991-1997.

Nurmi K and Kyngäs J (2011). Days-off scheduling for a bus transportation staff. International Journal of Innovative Computing and Applications 3(1):42-49.

Preux P and Talbi E-G (1999). Towards hybrid evolutionary algorithms. International Transactions in Operational Research 6(6):557-570.

Rasmussen P and Trick MA (2008). Round robin scheduling-a survey. European Journal of Operational Research 188(3): 617-636.

Trick MA (2001). A schedule-then-break approach to sports timetabling. In: Burke EK, Erben E (editors) The 3rd International Conference on the Practice and Theory of Automated Timetabling. Lecture Notes in Computer Science, vol 2079, pp 242-252.

Urban TL and Russell RA (2003). Scheduling sports competitions on multiple venues. European Journal of Operational Research 148(2):302-311.

Urban TL and Russell RA (2006). A constraint-programming approach to the multiple-venue, sport-scheduling problem. Computers and Operations Research 33(7):1895-1906.

Received 4 May 2016; accepted 18 October 2016 